\title{
ON THE INJECTIVITY OF THE ATTENUATED RADON TRANSFORM
}

\author{
ALEXANDER HERTLE
}

\begin{abstract}
We show that the attenuated (exponential) Radon transform $R_{\mu}$, where $\mu$ is assumed to be linear in the space variable, is injective on compactly supported distributions. Moreover, a limited angle reconstruction is possible and a hole theorem holds. We review the well-known special case of constant attenuation.
\end{abstract}

Introduction. Let $\mu \in C^{\infty}\left(S^{1} \times \mathbf{R}^{2}\right)$ be given. The attenuated Radon transform is the operator $R_{\mu}: C_{c}^{\infty}\left(\mathbf{R}^{2}\right) \rightarrow C_{c}^{\infty}\left(S^{1} \times \mathbf{R}\right)$, defined by

$$
R_{\mu} \varphi(\omega, p)=\int_{\omega \cdot x=p} \varphi(x) e^{\mu(\omega, x)} d x
$$

where $d x$ denotes the Lebesgue measure on the line $\omega \cdot x=p$. The adjoint $R_{\mu}^{t}: C^{\infty}\left(S^{1} \times \mathbf{R}\right) \rightarrow C^{\infty}\left(\mathbf{R}^{2}\right)$ of $R_{\mu}$ is given by

$$
R_{\mu}^{t} \psi(x)=\int_{S^{1}} \psi(\omega, \omega \cdot x) e^{\mu(\omega, x)} d \omega .
$$

As in the classical case $\mu \equiv 0$ (cf. [2]), we extend $R_{\mu}$ from $\mathcal{E}^{\prime}\left(\mathbf{R}^{2}\right)$ into $\mathcal{E}^{\prime}\left(S^{1} \times \mathbf{R}\right)$ via $\left\langle R_{\mu} u, \psi\right\rangle=\left\langle u, R_{\mu}^{t} \psi\right\rangle$, where $u \in \mathcal{E}^{\prime}\left(\mathbf{R}^{2}\right)$ and $\psi \in C^{\infty}\left(S^{1} \times \mathbf{R}\right)$.

The case $\mu(\omega, x)=$ (const) $\omega^{\perp} \cdot x$, where $\omega^{\perp}=\left(-\omega_{2}, \omega_{1}\right)$, is known as constant attenuation (see e.g. Tretiak and Metz [9]). In this note we assume throughout that $\mu$ is linear in $x$, i.e. there exists a $C^{\infty}$ vector field $\nu=\left(\nu_{1}, \nu_{2}\right): S^{1} \rightarrow \mathbf{R}^{2}$, such that

$$
\mu(\omega, x)=x \cdot \nu(\omega)=x_{1} \nu_{1}(\theta)+x_{2} \nu_{2}(\theta),
$$

where $\theta \in[0,2 \pi]$ and $\omega=(\cos \theta, \sin \theta)$. Thus $(0.2)$ can be regarded as a first order approximation to a general $\mu \in C^{\infty}$.

The results of this paper (except for the limited angle reconstruction) are known for constant attenuation. Tretiak and Metz [9] and Markoe [3] gave inversion formulae, and Quinto [6] proved the hole theorem in this case (his proof is not related to ours). Further, Quinto [6] inverted rotation invariant Radon transforms. But (0.1) is not rotation invariant, and in such cases only local invertibility results are known so far (Markoe and Quinto [4]).

Transforms of type (0.1) appear in problems pertaining to emission computed tomography, see e.g. Tretiak [8]. Of course, our injectivity result (§1) only demonstrates that a subclass (0.2) of such transforms can in principle be inverted. The limited angle theorem and the hole theorem (§2) are at least of theoretical interest (they are of even practical interest for the classical Radon transform).

Received by the editors October 20, 1983.

1980 Mathematics Subject Classification. Primary 44A05, 42B10.

Key words and phrases. Attenuated Radon transform, generalized Radon transform, Fourier transform.

(C) 1984 American Mathematical Society $0002-9939 / 84 \$ 1.00+\$ .25$ per page 
1. Injectivity and limited angle reconstruction. Let $u \in \mathcal{E}^{\prime}\left(\mathbf{R}^{2}\right)$ and $\mu$ as in (0.2). Then for $\omega \in S^{1}$ and $p \in \mathbf{R}$

$$
\hat{u}(p \omega+i \nu(\omega))=(\text { const })\left(R_{\mu} u\right)^{\Upsilon}(\omega, p),
$$

where $\hat{u}$ is the Fourier transform of $u$ and $\left(R_{\mu} u\right)^{\wedge}$ the Fourier transform of $R_{\mu} u$ w.r.t. the second variable. This projection theorem is verified as in the classical or constantly attenuated case (cf. Natterer [5]). Now let $I$ be a nonvoid open interval in $[0,2 \pi]$. Because of relation (1.1) we are interested in the surface

$$
M_{\mu}=\{z=p \omega+i \nu(\omega): p \in \mathbf{R}, \theta \in I\} \subset \mathbf{C}^{2},
$$

where again $\omega=(\cos \theta, \sin \theta)$. For $z \in M_{\mu}$ let $T_{z} M_{\mu}$ be the real-linear span of $\partial z / \partial p$ and $\partial z / \partial \theta$ :

$$
T_{z} M_{\mu}=\langle\partial z / \partial p, \partial z / \partial \theta\rangle .
$$

Since $\hat{u}$ is entire, by (1.1) injectivity of $R_{\mu}$ means essentially the existence of points $z$ in $M_{\mu}$ without complex tangents in $T_{z} M_{\mu}$, i.e. the complexification $T_{z} M_{\mu} \otimes \mathbf{C}$ of $T_{z} M_{\mu}$ is whole of $\mathbf{C}^{2}$. In particular, at such points, $\partial z / \partial p$ and $\partial z / \partial \theta$ form a basis of $\mathbf{C}^{2}$ (cf. $\left.[10, \S 17]\right)$.

Proposition 1.1. $M_{\mu}$ is a set of uniqueness for entire functions on $\mathbf{C}^{2}$. Indeed, if $z \in M_{\mu}$ with $p \neq 0$ then $\operatorname{dim}\left(T_{z} M_{\mu}\right)=2$ and

$$
T_{z} M_{\mu} \cap i T_{z} M_{\mu}=\{0\},
$$

i.e. the Taylor coefficients at $z$ of an entire function can be reconstructed from its values on a neighborhood of $z$ in $M_{\mu}$.

PROOF. First note that

$$
\partial z / \partial p=(\cos \theta, \sin \theta), \quad \partial z / \partial \theta=(-p \sin \theta, p \cos \theta)+i\left(\nu_{1}^{\prime}(\theta), \nu_{2}^{\prime}(\theta)\right) .
$$

Hence condition (1.3) gives rise to a homogeneous system of four linear equations, which matrix $A$ is given by

$$
A=\left(\begin{array}{cccc}
0 & -\nu_{1}^{\prime}(\theta) & \cos \theta & -p \sin \theta \\
0 & -\nu_{2}^{\prime}(\theta) & \sin \theta & p \cos \theta \\
\cos \theta & -p \sin \theta & 0 & \nu_{1}^{\prime}(\theta) \\
\sin \theta & p \cos \theta & 0 & \nu_{2}^{\prime}(\theta)
\end{array}\right) .
$$

The determinant of $A$ is immediately computed to

$$
\operatorname{det}(A)=p^{2}+\left(\nu_{2}^{\prime} \cos \theta-\nu_{1}^{\prime} \sin \theta\right)^{2},
$$

which means $\operatorname{det}(A) \neq 0$ if $p \neq 0$. This proves $\operatorname{dim}\left(T_{z} M_{\mu}\right)=2$ as well as equation (1.3) for $p \neq 0$. The rest of the proposition is now clear by the following remark.

REMARK. How can an entire function $f$ on $\mathbf{C}^{2}$ be recovered explicitly in the situation of Proposition 1.1?-To this purpose, let $z^{0} \in M_{\mu}$ and assume we know $f$ in a neighbourhood of $z^{0}$ on $M_{\mu}$. Then we know its partial derivatives in the (complex) directions $v=\partial z / \partial p$ and $w=\partial z / \partial \theta$, which (by Proposition 1.1) form a basis of $\mathbf{C}^{2}$. Let $A$ be a regular complex-linear map on $\mathbf{C}^{2}$, such that

$$
A v=(1,0) \text { and } A w=(0,1) .
$$


Applying Taylor's formula to the entire function $f \circ A^{-1}$, we obtain for $z \in \mathbf{C}^{2}$

$$
f\left(A^{-1} z\right)=\sum_{\alpha+\beta=0}^{\infty} \frac{1}{\alpha ! \beta !} \frac{\partial^{\alpha+\beta} f}{\partial^{\alpha} v \partial^{\beta} w}\left(z^{0}\right)\left(z_{1}-a_{1}\right)^{\alpha}\left(z_{2}-a_{2}\right)^{\beta}
$$

where $a=A z^{0} \in \mathbf{C}^{2}$. Thus we know $f$ everywhere on $\mathbf{C}^{2}$.

The following theorems are an easy consequence of Proposition 1.1.

THEOREM 1.2. $R_{\mu}: \mathcal{E}^{\prime}\left(\mathbf{R}^{2}\right) \rightarrow \mathcal{E}^{\prime}\left(S^{1} \times \mathbf{R}\right)$ is injective.

THEOREM 1.3 (LIMITED ANGLE THEOREM). If $u \in \mathcal{E}^{\prime}\left(\mathbf{R}^{2}\right)$ then $R_{\mu} u$ is determined by its values on any set $\Omega \times \mathbf{R}$, where $\Omega$ is nonvoid and open in $S^{1}$.

REMARK. For a submanifold $M$ of $\mathbf{C}^{2}$ and its tangent space $T_{z} M$, condition (1.3) is known as $M$ being totally real at $z$. In general we do not know that $M_{\mu}$ is a submanifold. But for constant attenuation a more precise description of $M_{\mu}$ is possible, see $\S 3$.

2. The hole theorem. Recall that $\mu$ is of the form (0.2), but for this section it suffices that $\nu: S^{1} \rightarrow \mathbf{R}^{2}$ is a $C^{1}$ vector field.-The proof of the following theorem is in its idea a variant of Helgason's proof for the classical case [2], of which a short presentation was given by Strichartz in [7].

THEOREM 2.1 (HOLE THEOREM). Let $\varphi$ be a Lipschitz continuous function of compact support on $\mathbf{R}^{2}$. Then for $r>0$

$$
\operatorname{supp}\left(R_{\mu} \varphi\right) \subset\{|p| \leq r\} \Rightarrow \operatorname{supp}(\varphi) \subset\{|x| \leq r\},
$$

where supp denotes the supports of the respective functions.

PROOF. We assume that $R_{\mu} \varphi$ is supported by $\{(\omega, p):|p| \leq r\}$. It suffices to show that $\varphi$ vanishes on every line $x_{1}=p$ with $p>r$; since if $U$ is a rotation, then

$$
R_{\mu} \varphi(U \omega, p)=R_{\mu^{\prime}} \varphi_{U}(\omega, p),
$$

where $\mu^{\prime}(\omega, x)=x \cdot U^{-1}(\nu(U \omega))$ and $\varphi_{U}(x)=\varphi(U x)$, i.e. $\mu^{\prime}$ is again of type (0.2). Thus it remains to prove

$$
\int t \varphi(p, t) \exp \left(p \nu_{1}(0)+t \nu_{2}(0)\right) d t=0
$$

then (by induction) we can replace the factor $t$ in (2.1) by any polynomial in $p$ and $t$, and the Weierstrass theorem finally gives $\varphi(p, \cdot) \equiv 0$. To show (2.1) let $U_{\theta}(p, t)=(p \cos \theta+t \sin \theta,-p \sin \theta+t \cos \theta)$ be a rotation by the angle $\theta$. By assumption we know

$$
\int \varphi\left(U_{\theta}(p, t)\right) \exp \left(U_{\theta}(p, t) \cdot \nu\left(e^{i \theta}\right)\right) d t=0
$$

for all $\theta$. Differentiating (2.2) with respect to $\theta$ and putting $\theta=0$ yields

$$
\begin{gathered}
\int \varphi(p, t) \exp \left(p \nu_{1}(0)+t \nu_{2}(0)\right)\left(p \nu_{1}^{\prime}(0)+t \nu_{2}^{\prime}(0)+t \nu_{1}(0)-p \nu_{2}(0)\right) d t \\
+\int(t \partial \varphi / \partial p-p \partial \varphi / \partial t) \exp \left(p \nu_{1}(0)+t \nu_{2}(0)\right) d t=0 .
\end{gathered}
$$


Since $\varphi$ is Lipschitz continuous, integration by parts shows

$$
-\int \partial \varphi / \partial t \exp \left(p \nu_{1}(0)+t \nu_{2}(0)\right) d t=0
$$

using (2.2) with $\theta=0$. Applying (2.2) also to the first integral in (2.3) we obtain therefore

$$
\begin{gathered}
\int \varphi(p, t) \exp \left(p \nu_{1}(0)+t \nu_{2}(0)\right) t\left(\nu_{2}^{\prime}(0)+\nu_{1}(0)\right) d t \\
+\int t \partial \varphi / \partial p \exp \left(p \nu_{1}(0)+t \nu_{2}(0)\right) d t=0 .
\end{gathered}
$$

We put $a=\nu_{2}^{\prime}(0)+\nu_{1}(0)$ and divide by $\exp \left(p \nu_{1}(0)\right)$ in (2.4). Thus

$$
(a+\partial / \partial p) \int t \varphi(p, t) \exp \left(t \nu_{2}(0)\right) d t \equiv 0
$$

for $p>r$. But this implies

$$
\int t \varphi(p, t) \exp \left(t \nu_{2}(0)\right) d t=(\text { const }) e^{-a p}
$$

for all $p>r$. Since $\varphi$ has compact support, the integral in (2.5) must vanish for large $p$, i.e. const $=0$. This shows (2.1) and the theorem is proved.

REMARK 2.2. Theorem 2.1 also implies the injectivity of $R_{\mu}$ on $C_{c}^{1}\left(\mathbf{R}^{2}\right)$. Our proof does not show directly whether the hole theorem holds on $\mathcal{E}^{\prime}\left(\mathbf{R}^{2}\right)$ for $R_{\mu}$ (as in the classical [2] or rotation invariant [6] case).

3. The case of constant attenuation. We finally study $M_{\mu}$ (cf. $\S 1$ ) in the case of constant attenuation. A precise description of $M_{\mu}$ is possible then, and if $\mu \neq 0$ the location of $M_{\mu}$ in $\mathbf{C}^{2}$ shows an interesting difference to the classical situation $M_{\mu}=\mathbf{R}^{2}$. As usual, for $z=\left(z_{1}, z_{2}\right) \in \mathbf{C}^{2}$ we write $z_{1}=x_{1}+i x_{3}$ and $z_{2}=x_{2}+i x_{4}$.

THEOREM 3.1. If $\mu(\omega, x)=c x \cdot \omega^{\perp}, c \neq 0$, then $M_{\mu}$ is an algebraic submanifold of $\mathbf{C}^{2}$, given by the equations

$$
x_{3}^{2}+x_{4}^{2}-c^{2}=0 \quad \text { and } \quad x_{1} x_{3}+x_{2} x_{4}=0 .
$$

$A$ point $z \in M_{\mu}$ is not totally real if and only if $x_{1}=x_{2}=0$, i.e. if $p=0$ in (1.2).

PROOF. The proof is straightforward if we observe that a point of a manifold $M=\left\{\rho_{1}(x)=\rho_{2}(x)=0\right\}$ is totally real if and only if $\operatorname{det}\left(\partial \rho_{m} / \bar{\partial} z_{j}\right) \neq 0$ at this point, see Cirka [1]. In fact, in our case we have from (3.1) that $\operatorname{det}\left(\partial \rho_{m} / \bar{\partial} z_{j}\right)=$ $c p / 2$.

In particular, if $c \neq 0, M_{\mu}$ contains points $z$ at which not all Taylor coefficients of an entire function on $\mathbf{C}^{2}$ can be reconstructed (from a neighbourhood of $z$ in $\left.M_{\mu}\right)$.

ACKNOWLEDGement. This paper was prepared during a stay at Tufts University, Medford. I would like to thank the Mathematics Department and Professor Quinto, in particular, for their kind hospitality.

ADDED IN PROOF. Professor F. Natterer informed me that his group at Münster obtained Theorem 1.2 by other methods. 


\section{REFERENCES}

1. E. M. Cirka, Approximation of holomorphic functions on smooth manifolds in $C^{n}$, Math. USSR Sb. 7 (1969), 95-114.

2. S. Helgason, The Radon transform, Birkhäuser, Boston, Mass., 1980.

3. A. Markoe, Fourier inversion of the attenuated X-ray transform, SIAM J. Math. Anal. (to appear).

4. A. Markoe and E. T. Quinto, An elementary proof of local invertibility for generalized and attenuated Radon transforms, Preprint 1983.

5. F. Natterer, On the inversion of the attenuated Radon transform, Numer. Math. 32 (1979), 431438.

6. E. T. Quinto, The invertibility of rotation invariant Radon transforms, J. Math. Anal. Appl. 91 (1983), 510-522.

7. R. S. Strichartz, Radon inversion-variations on a theme, Amer. Math. Monthly 89 (1982), 377-384.

8. O. J. Tretiak, Attenuated and exponential Radon transforms, Proc. Sympos. Appl. Math., vol. 27, Amer. Math. Soc., Providence, R.I., 1982.

9. O. J. Tretiak and C. L. Metz, The exponential Radon transform, SIAM J. Appl. Math. 39 (1980), 341-354.

10. J. Wermer, Banach algebras and several complex variables, 2nd ed., Springer, Berlin and New York, 1976.

FACHBEREich Mathematik, Universität Mainz, SAARStrasse 21, D-6500 Mainz, FEDERAL REPUBLIC OF GERMANY 